\title{
On a Geometric Realization of $\mathcal{A}(\mathbf{2})$
}

\author{
By
}

\author{
Kouichi INOUE*
}

\section{$\S 0$. Introduction}

Let $A$ be the $\bmod p$ Steenrod algebra and $M$ be a bounded below left $A$ module of finite type. $M$ is said to be realizable if there exists some spectrum whose $\bmod p$ cohomology is isomorphic as $\mathcal{A}$-module to $\mathrm{M}$. For example, $\mathcal{A}$ itself is realized as $\mathcal{A} \cong \mathrm{H}^{*}(\mathrm{HZ} / p ; Z / p)$. It is a general problem whether or not given $\mathrm{M}$ is realizable, but there is no standard method to solve this problem. So we have to try case by case. For many interesting cases, this problem was solved. J.F. Adams [1] showed that there is no spectrum which realizes $\mathrm{M} \cong$ $Z / 2 \cdot x+Z / 2 \cdot S q^{16} x$. E. H. Brown and S. Gitler [2] constructed certain spectra $\mathrm{B}(k)$ such that $\mathrm{H} * \mathrm{~B}(k) \cong \mathcal{A} / \mathcal{A}\left\{\chi\left(S q^{i}\right) \mid i>k\right\}$. H. Toda [8] stated that certain algebraic properties of $\mathrm{M}$ assure its realizability. In this paper we shall prove that some more conditions give us useful information about the number of the homotopy types of spectra which realize M. (Theorem 1.1)

$\mathcal{A}(n)$ is a sub-Hopf algebra of $\mathcal{A}$ generated by $\beta, \mathscr{Q}^{1}, \cdots, \mathscr{Q}^{p^{n-1}}$, with $\mathscr{P}^{i}=$ $S q^{2 i}$ for $p=2$. S. A. Mitchell [6] proved every $\mathcal{A}(n)$ admits certain left $\mathcal{A}$ module structure extended from its own algebra multiplication and also constructed finite spectra whose cohomologies are $\mathcal{A}(n)$ free. Hence we should ask whether each $\mathcal{A}(n)$ itself is realizable or not, because there exists a non-realizable $\mathcal{A}$-module which is a direct summand of a realizable module.

Independently of Mitchell's work, D. M. Davis and M. Mahowald [3] gave four different module structures on $\mathcal{A}(1)(p=2)$ and proved the uniqueness of the homotopy type of spectra which realize each $\mathcal{A}(1)$. For the case of $\mathcal{A}(2)$ $(p=2), W . H$. Lin [4] showed 1600 different $\mathcal{A}$-module structures. Theorem 2.2 gives an affirmative answer to the realization problem for $\mathcal{A}(2)(p=2)$ with any possible $A$-module structure and Theorem 2.4 shows the uniqueness of the homotopy type of spectra which realize $\mathcal{A}(2)$ with the specific $A$-module structure indicated by Mitchell [6].

Communicated by N. Shimada, March 2, 1988.

* Department of Mathematics, Faculty of Science, Kyoto Unıversity, Kyoto 606, Japan. 


\section{$\S 1$. The Number of the Homotopy Types}

We work in the homotopy category of $\mathrm{HZ} / p_{*}$-local $\mathrm{CW}$-spectra, because any spectrum $\boldsymbol{X}$ has the same $\bmod p$ cohomology group as its $\mathrm{HZ} / p_{*}$-localization $\overline{\boldsymbol{X}}$, cohomology equivalence means (homotopy) equivalence and this category includes usual bounded below $p$-complete spectra. In larger categories, it might be impossible to count the homotopy types of spectra which realize the same $A$ module because of the existence of $\mathrm{HZ} / p_{*}$-acyclic spectra.

Theorem 1.1. Let $\mathrm{M}$ be a bounded below A-module of finite type with the following properties:

(1) $\mathrm{M}^{n} \neq 0$ implies $\mathrm{Ext}_{\mathcal{U}}^{s+2, s+n}\left(\mathrm{M}, \boldsymbol{F}_{p}\right)=0$ for $s \geqq 1$,

(2) $\mathrm{M}^{n} \neq 0$ implies $\operatorname{Ext}_{\mathcal{A}}^{s+1, s+n}\left(\mathrm{M}, \boldsymbol{F}_{p}\right)=0$ for $s \geqq 2$.

Then there exists a bounded below $\mathrm{HZ} / p_{*}$-local spectrum $\boldsymbol{X}$ such that $\mathrm{M} \cong \mathrm{H}^{*} \boldsymbol{X}$ as A-module. And let $\Sigma$ be the set of the homotopy types of such spectra, then the following inequalities hold: (Here $|\Sigma|$ means the number of the elements of ¿.)

$$
\left|\operatorname{Ext}_{\circlearrowleft !}^{2,1}(M, M)\right| /\left|\operatorname{Aut}_{\mathcal{A}}(\mathrm{M})\right| \leqq|\Sigma| \leqq\left|\operatorname{Ext}_{\mathcal{J}}^{2,1}(\mathrm{M}, \mathrm{M})\right|
$$

Proof. The existence of such a spectrum follows from Toda [8] by only using the condition (1). We recall it for the further proof.

Fix a minimal resolution of $\mathrm{M}$ as $\mathcal{A}$-module:

$$
0 \longleftarrow \mathrm{M} \longleftarrow \mathrm{C}^{0} \stackrel{\delta^{1}}{\longleftarrow} \mathrm{C}^{1} \stackrel{\delta^{2}}{\longleftarrow} \mathrm{C}^{2} \stackrel{\delta^{3}}{\longleftarrow} \cdots, \text { where } \mathrm{C}^{s} \cong \mathcal{A} \otimes \operatorname{Ext}_{\mathcal{H}}^{s, *}\left(\mathrm{M}, \boldsymbol{F}_{p}\right) .
$$

$\mathrm{C}^{s}$ is realized by a generalized Eilenberg-MacLane spectrum $W_{s}$. And starting from $\boldsymbol{X}_{0}=\boldsymbol{W}_{0}$, we can construct a sequence of spectra $\left\{\boldsymbol{X}_{s}\right\}$ satisfying the following conditions:

a) There are fibrations $\Sigma^{-s} \boldsymbol{W}_{s} \stackrel{k_{s}}{\rightarrow} \boldsymbol{X}_{s} \stackrel{i_{s}}{\rightarrow} \boldsymbol{X}_{s-1} \stackrel{\pi_{s}}{\rightarrow} \Sigma^{-s+1} \boldsymbol{W}_{s}$ which induce exact sequences :

$$
\mathrm{C}^{s, s+n} \stackrel{k_{s}^{*}}{\longleftarrow} \mathrm{H}^{n} \boldsymbol{X}_{s} \stackrel{i_{s}^{*}}{\longleftarrow} \mathrm{H}^{n} \boldsymbol{X}_{s-1} \stackrel{\pi_{s}^{*}}{\longleftarrow} \mathrm{C}^{s, s+n-1} .
$$

b) $\delta^{s+1}=k_{s}^{*} \circ \pi_{s+1}^{*}: \mathrm{C}^{s+1} \rightarrow \mathrm{H}^{*} \boldsymbol{X}_{s} \rightarrow \mathrm{C}^{s}$.

c) There are split short exact sequences:

$$
0 \longrightarrow \mathrm{M} \longrightarrow \mathrm{H}^{*} \boldsymbol{X}_{s} \stackrel{k_{s}^{*}}{\longrightarrow} \delta^{s+1}\left(\mathrm{C}^{s+1}\right) \longrightarrow 0 .
$$

Then the spectrum $\boldsymbol{X}=\lim \boldsymbol{X}_{s}$ realizes M. 
$(*)$

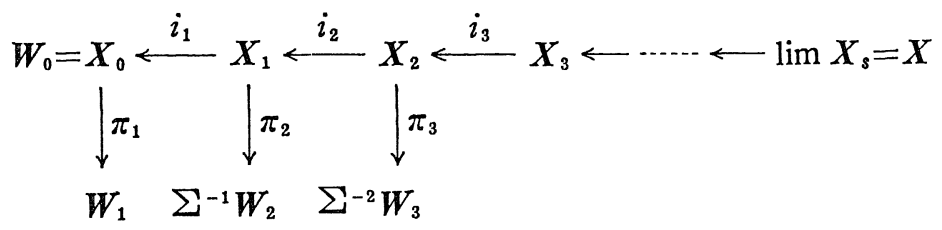

Next we prove any spectrum realizing $M$ is homotopy equivalent to some spectrum obtained by the above method. Let $\boldsymbol{Y}$ be such a spectrum, and $g_{0}: \boldsymbol{Y}$ $\rightarrow \boldsymbol{X}_{0}$ be a map realizing $\varepsilon: \mathrm{C}^{0}=\mathrm{H}^{*} \boldsymbol{X}_{0} \rightarrow \mathrm{M} \cong \mathrm{H}^{*} \boldsymbol{Y}$. Since $\pi_{1} \circ g_{0} \simeq 0, g_{0}$ has a lift $g_{1}: Y \rightarrow X_{1}$. Moreover there exists a map $\alpha: X_{0} \rightarrow \Sigma^{-1} W_{2}$ such that $\alpha \circ g_{0} \simeq \pi_{2} \circ g_{1}$, because $\varepsilon$ is surjective. Even if we replace $\pi_{2}$ by $\pi_{2}^{\prime}=\pi_{2}-\alpha \circ i_{1}$, we can proceed the construction of another sequence of spectra $\left\{X_{s}^{\prime}\right\}$ from which $\boldsymbol{X}^{\prime}=\lim \boldsymbol{X}_{s}^{\prime}$ also realizes $M$, because each $\pi_{s}$ is required only to satisfy the above condition b).

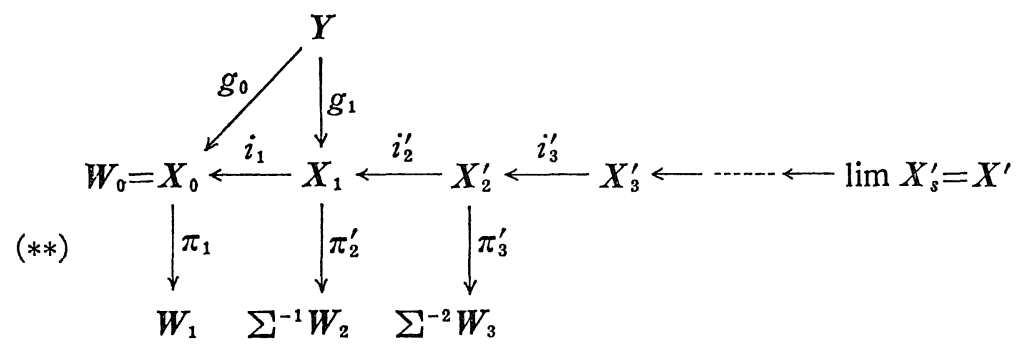

Now consider the Adams spectral sequence associated to this tower (**):

$$
\operatorname{Ext}_{\AA}^{* *}\left(\mathrm{H}^{*} \boldsymbol{X}^{\prime}, \mathrm{H}^{*} \boldsymbol{Y}\right) \Longrightarrow\left[\boldsymbol{Y}, \boldsymbol{X}^{\prime}\right]^{*} .
$$

Since we fixed a map $g_{0}: Y \rightarrow X_{0}$ realizing $\varepsilon: \mathrm{C}^{0} \rightarrow \mathrm{M}$, there is one and only one isomorphism $\beta: \mathrm{H}^{*} \boldsymbol{X}^{\prime} \rightarrow \mathrm{H}^{*} \boldsymbol{Y}$ such that $g_{0}^{*}=\beta \circ f_{0}^{\prime *}$. ( $f_{s}^{\prime}$ is a composition of maps $\left.\boldsymbol{X}^{\prime} \rightarrow \cdots \rightarrow \boldsymbol{X}_{s+1}^{\prime} \rightarrow \boldsymbol{X}_{s}^{\prime}\right)$ And $\beta \in \mathrm{E}_{2}^{0,0}=\mathrm{Hom}_{\mathfrak{A}}\left(\mathrm{H}^{*} \boldsymbol{X}^{\prime}, \mathrm{H}^{*} \boldsymbol{Y}\right)$ is represented by $g_{0}$ in the spectral sequence. Since $\pi_{2}^{\prime} \circ g_{1}=\pi_{2} \circ g_{1}-\alpha \circ i_{1} \circ g_{1}=\pi_{2} \circ g_{1}-\alpha \circ g_{0}=0 \in$ $\left[Y, \Sigma^{-1} W_{2}\right]$, we get $d_{2}(\beta)=0 \in \mathrm{E}_{2}^{2,1}$. And the condition (2) implies:

$$
\operatorname{Hom}_{\mathfrak{A}}^{s}\left(\mathrm{C}^{s+1}, \mathrm{M}\right) \cong \operatorname{Hom}_{\mathfrak{A}}^{s}\left(\mathcal{A} \otimes \operatorname{Ext}_{\mathcal{A}}^{s+1,} *\left(\mathrm{M}, \boldsymbol{F}_{p}\right), \mathrm{M}\right) \cong \operatorname{Hom}^{s}\left(\operatorname{Ext}_{\mathfrak{A}}^{s+1, *}\left(\mathrm{M}, \boldsymbol{F}_{p}\right), \mathrm{M}\right) \cong 0
$$

for $s \geqq 2$. So $\operatorname{Ext}_{\mathcal{H}}^{s+1, s}(\mathrm{M}, \mathrm{M}) \cong 0$, that is, $d_{s+1}(\beta)=0$. Thus there exists a map $g: Y \rightarrow X^{\prime}$ realizing $\beta$. Since $X^{\prime}$ and $Y$ are HZ/ $p_{*}$-local spectra, $g$ is a homotopy equivalence.

Next we construct a set function $\Phi$ which has $\Sigma$ as its domain and the set of subsets of $\operatorname{Ext}_{M}^{2,1}(\mathrm{M}, \mathrm{M})$ as its target. As studied above, the isomorphism $\beta^{\prime}: M=H^{*} \boldsymbol{X} \rightarrow \mathrm{H}^{*} \boldsymbol{X}^{\prime}$ is uniquely determined for any $\boldsymbol{X}^{\prime} \in \Sigma$ and any map $f_{0}^{\prime}: \boldsymbol{X}^{\prime}$ $\rightarrow \boldsymbol{W}_{0}$ realizing $\varepsilon$. We consider the Adams spectral sequence $\mathrm{E}_{2}^{* *} \cong \operatorname{Ext}_{*}^{* *}\left(\mathrm{H}^{*} \boldsymbol{X}, \mathrm{H}^{*} \boldsymbol{X}^{\prime}\right)$ $\Rightarrow\left[\boldsymbol{X}^{\prime}, \boldsymbol{X}\right]^{*}$ associated to the tower $(*)$, and put 


$$
\begin{aligned}
& \Phi\left(X^{\prime}, f_{0}^{\prime}\right)=\beta_{*}^{\prime-1}\left(d_{2}\left(\beta^{\prime}\right)\right) \in \operatorname{Ext}_{\mathfrak{d}}^{2,1}(\mathrm{M}, \mathrm{M}) . \\
& \operatorname{Hom}_{\mathfrak{A}}\left(\mathrm{H}^{*} \boldsymbol{X}, \mathrm{H}^{*} \boldsymbol{X}^{\prime}\right) \stackrel{d_{2}}{\longrightarrow} \operatorname{Ext}_{\mathfrak{\sharp}}^{2,1}\left(\mathrm{H}^{*} \boldsymbol{X}, \mathrm{H}^{*} \boldsymbol{X}^{\prime}\right) \stackrel{\beta_{*}^{\prime-1}}{\longrightarrow} \operatorname{Ext}_{\mathfrak{l}}^{2,1}(\mathrm{M}, \mathrm{M})
\end{aligned}
$$

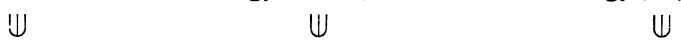

$$
\begin{aligned}
& \beta^{\prime} \longmapsto d_{2}\left(\beta^{\prime}\right) \longmapsto \Phi\left(X^{\prime}, f_{0}^{\prime}\right)
\end{aligned}
$$

Let $\Phi\left(\mathbb{X}^{\prime}\right)=\left\{\Phi\left(\mathbb{X}^{\prime}, f_{0}^{\prime}\right) \mid\right.$ for all possible $\left.f_{0}^{\prime \prime} s\right\}$, then $\Phi$ has a property such that $\Phi\left(\boldsymbol{X}^{\prime}\right) \cap \Phi\left(\boldsymbol{X}^{\prime \prime}\right) \neq \phi$ implies $\boldsymbol{X}^{\prime} \cong \boldsymbol{X}^{\prime \prime}$. To see this, suppose $\Phi\left(\boldsymbol{X}^{\prime}, f_{0}^{\prime}\right)=$ $\Phi\left(X^{\prime \prime}, f_{0}^{\prime \prime}\right)$, then from the above diagram,

$$
\beta_{*}^{\prime} \circ \beta_{*}^{\prime \prime-1}\left[\pi_{2} \circ f_{1}^{\prime \prime}\right]=\left[\pi_{2} \circ f_{1}^{\prime}\right] \in \operatorname{Ext}_{\mathcal{H}}^{2,1}\left(\mathrm{H}^{*} \boldsymbol{X}, \mathrm{H}^{*} X^{\prime}\right) .
$$

Ext $^{2,1}$ is defined as the (co)homology of sequence:

$$
\begin{aligned}
& \operatorname{Hom}_{\lrcorner}^{1}\left(\mathrm{C}^{1}, \mathrm{H}^{*} X^{\prime}\right) \stackrel{\delta^{2 *}}{\longrightarrow} \operatorname{Hom}_{\mathfrak{l}}^{1}\left(\mathrm{C}^{2}, \mathrm{H}^{*} X^{\prime}\right) \stackrel{\delta^{3 *}}{\longrightarrow} \operatorname{Hom}_{\mathfrak{A}}^{1}\left(\mathrm{C}^{3}, \mathrm{H}^{*} X^{\prime}\right) \cong 0, \\
& \begin{array}{cc}
\uparrow \cong & \uparrow \cong \\
{\left[X^{\prime}, \Sigma^{-1} W_{1}\right]} & {\left[X^{\prime}, \Sigma^{-1} W_{2}\right]}
\end{array}
\end{aligned}
$$

where the last isomorphism is due to the condition (1), and the vertical isomorphisms mean sending a map between spectra to its induced map between their cohomologies.

So there exists a map $h: X^{\prime} \rightarrow \Sigma^{-1} W_{1}$ such that

$$
f_{1}^{\prime *} \circ \pi_{2}^{*}-\beta^{\prime} \circ \beta^{\prime \prime-1} \circ f_{1}^{\prime \prime *} \circ \pi_{2}^{*}=h^{*} \circ \delta^{2} \quad \text { in } \operatorname{Hom}_{\mathfrak{l}}^{1}\left(\mathrm{C}^{2}, \mathrm{H}^{*} \boldsymbol{X}^{\prime}\right) .
$$

And by taking maps $\alpha^{\prime}, \alpha^{\prime \prime}: X_{0} \rightarrow \Sigma^{-1} W_{2}$ such that

$$
\pi_{2} \circ f_{1}^{\prime}=\alpha^{\prime} \circ f_{0}^{\prime}, \quad \pi_{2} \circ f_{1}^{\prime \prime}=\alpha^{\prime \prime} \circ f_{0}^{\prime},
$$

we get the following equation:

$$
f_{0}^{\prime *} \circ\left(\alpha^{*}-\alpha^{\prime \prime *}\right)=h^{*} \circ \delta^{2} 。
$$

Again consider the Adams spectral sequence associated to the tower (**):

$$
\begin{aligned}
& \mathrm{E}_{2}^{* *} \cong \operatorname{Ext}_{\lrcorner}^{* *}\left(\mathrm{H}^{*} X^{\prime \prime}, \mathrm{H}^{*} \boldsymbol{X}^{\prime}\right) \Longrightarrow\left[\boldsymbol{X}^{\prime}, \boldsymbol{X}^{\prime \prime}\right]^{*} . \\
& d_{2}\left(\boldsymbol{\beta}^{\prime} \circ \beta^{\prime \prime-1}\right)=\left[\left(\pi_{2}^{\prime \prime} \circ f_{1}^{\prime}\right)^{*}\right]=\left[f_{0}^{\prime *} \circ\left(\alpha^{*}-\alpha^{\prime \prime}\right)\right]=\left[h^{*} \circ \delta^{2}\right]=0 .
\end{aligned}
$$

Therefore $\beta^{\prime} \circ \beta^{\prime \prime-1}$ is realizable, namely, $X^{\prime} \simeq X^{\prime \prime}$.

On the other hand, for any $\left[\alpha \circ f_{0}\right] \in \operatorname{Ext}_{\AA}^{2,1}(\mathrm{M}, \mathrm{M})$, we can construct a spectrum $X^{\prime}$ from $\pi_{2}^{\prime}=\pi_{2}-\alpha \circ i_{1}$. Then $\Phi\left(X^{\prime}, f_{0}^{\prime}\right)=\beta^{-1} *\left[\pi_{2} \circ f_{1}^{\prime}\right]=\left[\alpha \circ f_{0}\right]$. Thus we can conclude:

$$
\underset{x \in \Sigma}{\Perp} \Phi(X)=\operatorname{Ext}_{\mathfrak{A}}^{2,1}(\mathrm{M}, \mathrm{M}),
$$

where $\Perp$ means disjoint union.

Suppose the following situation: $H^{*} X^{\prime} \underset{\beta_{2}^{\prime}}{\stackrel{\cong}{\stackrel{\cong}{\beta_{1}^{\prime}}}} \stackrel{\cong}{\cong} H^{*} X^{\prime}$. As studied above, 
$\beta_{1}^{\prime-1} * d_{2}\left(\beta_{1}^{\prime}\right)=\beta_{2}^{\prime-1} * d_{2}\left(\beta_{2}^{\prime}\right)$ iff $\beta_{1}^{\prime} \circ \beta_{2}^{\prime-1}$ is realizable. Thus there is a one-to-one correspondence between $\Phi\left(\boldsymbol{X}^{\prime}\right)$ and $\operatorname{Heq}\left(\boldsymbol{X}^{\prime}\right) \backslash \mathrm{Aut}_{\mathfrak{A}}\left(\mathrm{H}^{*} \boldsymbol{X}^{\prime}\right)$, where Heq $\left(\boldsymbol{X}^{\prime}\right)$ is a subgroup of Aut $_{A}\left(\mathrm{H}^{*} \boldsymbol{X}^{\prime}\right)$ whose elements are induced from self homotopy equivalences on $\boldsymbol{X}^{\prime}$. Then the inequalities in the theorem follow easily. (Q.E.D.)

Remark. It is very hard to calculate $\left|\mathrm{Heq}\left(\boldsymbol{X}^{\prime}\right)\right|$ and, what is worse, $\mid$ Heq $\left(\boldsymbol{X}^{\prime}\right) \mid$ may be different for each $\boldsymbol{X}^{\prime}$. So I had to find satisfaction in these inequalities, regrettably.

\section{$\S 2$. Realization of $\mathcal{A}(2)(p=2)$}

Milnor basis of $\mathcal{A}$ is an $\boldsymbol{F}_{2}$ vector space basis of $\mathcal{A}$ written as $\left\{S q\left(r_{1}, r_{2}, r_{3}, \cdots\right) \mid r_{i} \geqq 0\right\}$. See J. Milnor [5] for the further structures. Using this notation, we can define $\mathcal{A}(n)$ as a vector subspace of $\mathcal{A}$ whose basis is $\left\{S q\left(r_{1}, r_{2}, \cdots, r_{n+1}\right) \mid 0 \leqq r_{i}<2^{n+2-i}\right\} . \quad \mathrm{P}_{t}^{s}$ is defined as $S q\left(0, \cdots, 0,2^{s}\right)$, where $2^{s}$ is occured in the $t$-th entry.

We consider the reindexed version of the May spectral sequence for $\operatorname{Ext}_{\mathcal{A}}^{* *}\left(\mathcal{A}(2), \boldsymbol{F}_{2}\right)$ according to D. C. Ravenel [7]:

$$
\begin{aligned}
& \mathrm{E}_{1}^{s, t, u} \cong \mathrm{Ext}_{\mathrm{E}_{0}, t}^{s, t, u}\left(\mathrm{E}_{0} \mathcal{A}(2), \boldsymbol{F}_{2}\right) \Longrightarrow \operatorname{Ext}_{\mathcal{H}}^{s, t}\left(\mathcal{A}(2), \boldsymbol{F}_{2}\right), \\
& d_{r}: \mathrm{E}_{r}^{s, t, u} \longrightarrow \mathrm{E}_{r}^{s+1, t, u+1-2 r} .
\end{aligned}
$$

Here, $E_{0} \mathcal{A}$ is the bigraded Hopf algebra associated with a decreasing filtration on $A$ defined by setting $\left|\mathrm{P}_{t}^{s}\right|=2 t-1$. In fact:

$$
\mathrm{E}_{0} \mathcal{A} \cong \mathrm{E}\left(\mathrm{P}_{t}^{s} ; t>0, s \geqq 0\right), \quad \mathrm{P}_{t}^{s}: \text { primitive. }
$$

Lemma 2.1. $\mathcal{A}(2)$ has 1600 different $A$-module structures (Lin [4]), but every $\mathrm{E}_{0} \mathcal{A}(2)$ has the same $\mathrm{E}_{0} \mathcal{A}$ module structure such that:

$$
\mathrm{E}_{0} \mathcal{A}(2) \cong \mathrm{E}\left(\mathrm{P}_{t}^{s} ; t>0, s \geqq 0, t+s \leqq 3\right) .
$$

Proof. $A$ has a free $\mathcal{A}(2)$ basis $\left\{\mathrm{P}_{1}^{3}, \mathrm{P}_{2}^{2}, \mathrm{P}_{3}^{1}, \mathrm{P}_{4}^{0}, \mathrm{P}_{1}^{4}\right\}$ up to degree 23 , the maximal degree of $\mathcal{A}(2)$. So weh ave only to show these are mapped into higher filtration when they are applied to $\iota$, the fundamental class of $\mathcal{A}(2)$. But this is immediate because $\mathcal{A}(2)$ has only such higher filtration degree elements in the degree of $\mathrm{P}_{t}^{s}$.

$$
\begin{aligned}
& \left|\mathrm{P}_{1}^{3}\right|=1, \quad|S q(5,1)|=5,|S q(2,2)|=4, \quad|S q(1,0,1)|=6, \\
& \left|\mathrm{P}_{2}^{2}\right|=3, \quad|S q(6,2)|=5,|S q(3,3)|=8, \quad|S q(5,0,1)|=7, \quad|S q(2,1,1)|=9 \text {, } \\
& \left|\mathrm{P}_{3}^{1}\right|=5, \quad|S q(5,3)|=8,|S q(7,0,1)|=8, \quad|S q(4,1,1)|=9, \quad|S q(1,2,1)|=9, \\
& \left|\mathrm{P}_{4}^{0}\right|=7, \quad|S q(6,3)|=8,|S q(5,1,1)|=10,|S q(2,2,1)|=9 \text {, } \\
& \left|\mathrm{P}_{1}^{4}\right|=1, \quad|S q(7,3)|=9,|S q(6,1,1)|=10,|S q(3,2,1)|=10,|S q(0,3,1)|=11 \text {. }
\end{aligned}
$$


Theorem 2.2. For any $\mathcal{A}$-module structure, $\mathcal{A}(2)$ is realizable.

Proof. $\quad \operatorname{Ext}_{\mathrm{E}_{0} \mathcal{H}}^{* * *}\left(\boldsymbol{F}_{2}, \boldsymbol{F}_{2}\right) \cong \mathrm{P}\left(h_{i, j} ; i>0, j \geqq 0\right)$,

where $\operatorname{deg} h_{\imath, j}=\left(1,2^{j}\left(2^{i}-1\right), 2 i-1\right)$. Each $h_{\imath, j}$ is represented by $\left[\mathrm{P}_{\imath}^{j}\right]$ in the bar complex. The above lemma and change-of-rings isomorphism induce:

$$
\begin{aligned}
\operatorname{Ext}_{\mathrm{E}_{0} \mathcal{H}}^{* * *}\left(\mathrm{E}_{0} \mathcal{A}(2), \boldsymbol{F}_{2}\right) & \cong \operatorname{Ext}_{\mathrm{E}}^{* * *}\left(\boldsymbol{F}_{2}, \boldsymbol{F}_{2}\right) \\
& \cong \mathrm{P}\left(h_{i, j}^{\prime} ; i>0, j \geqq 0, i+j>3\right),
\end{aligned}
$$

where $\mathrm{E}=\mathrm{E}\left(\mathrm{P}_{t}^{s} ; t>0, s \geqq 0, t+s>3\right)$ and $h_{i, j}^{\prime}$ is an image of $h_{i, j}$ through the map $\mathrm{E}_{0} \mathcal{A}(2) \rightarrow \boldsymbol{F}_{2}$.

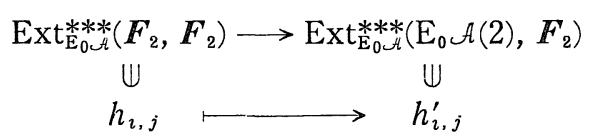

In the $\mathrm{E}_{1}$-term, there is one element which might survive in the $\mathrm{E}_{\infty}$-term and give some non-zero element of $\operatorname{Ext}_{\mathscr{A}}^{s+2, s+n}\left(\mathcal{A}(2), \boldsymbol{F}_{2}\right)$ for $s \geqq 1,0 \leqq n \leqq 23$. To say precisely, since $\operatorname{deg} h_{1,3}^{\prime}=(1,8,1), h_{1,3}^{\prime s+2}$ is the lowest degree element in $\{x \mid \operatorname{deg} x=(s+2, *, *)\}$. But $\operatorname{deg} h_{1,3}^{\prime s+2}=8(s+2)>s+23$ for $s>1$, so the element mentioned above is $h_{1,3}^{\prime}{ }^{3}$.

$d_{2}\left(h_{2,2}^{\prime}{ }^{2}\right)=h_{1,3}^{\prime}{ }^{3}$, however, because the corresponding differential in the May spectral sequence for $\operatorname{Ext}_{\mathbb{A}}^{* *}\left(\boldsymbol{F}_{2}, \boldsymbol{F}_{2}\right)$ is

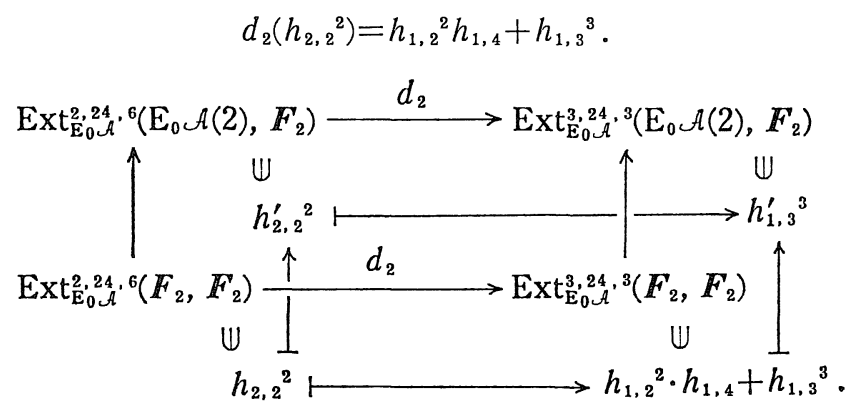

Therefore for any $A$-module structure on $\mathcal{A}(2)$, we can conclude $\operatorname{Ext}_{\mathcal{H}}^{s+2, s+n}\left(\mathcal{A}(2), \mathbb{F}_{2}\right) \equiv 0$ for $s \geqq 1,0 \leqq n \leqq 23$, that is, $\mathcal{A}(2)$ is realizable. (Q. E. D.)

Note. We cannot proceed the same approach as the above for the realization of $\mathcal{A}(n)(n>2)$, because there might survive many elements in the $\mathrm{E}_{\infty}$-term so as to generate obstructions in $\operatorname{Ext}_{\mathcal{H}}^{s+2, s+m}\left(\mathcal{A}(n), \boldsymbol{F}_{2}\right)$ for $s \geqq 1,0 \leqq m \leqq$ maxdeg $\mathcal{A}(n)$. For example, $h_{1,4}^{\prime}{ }^{2} \cdot h_{5,0}^{\prime} \in \operatorname{Ext}_{\mathrm{E}_{0} A^{\prime}}^{3,63,11}\left(\mathrm{E}_{0} \mathcal{A}(3), \boldsymbol{F}_{2}\right)$ is a permanent cycle, because there exists no element in $\mathrm{E}_{1}^{2,63, *}$ whose filtration degree is greater than 11 , and $\mathrm{E}_{1}^{4, t}, * \equiv 0$ for $t<64$.

Next we will prove the uniqueness of the homotopy type of spectra which 
realize $A(2)$ with the specific $A$-module structure indicated by Mitchell [6]. I calculated in my master thesis its explicit presentation form as follows.

Proposition 2.3. $0 \leftarrow \mathcal{A}(2) \stackrel{\varepsilon}{\leftarrow} A \stackrel{\delta^{1}}{\leftarrow} \mathrm{C}^{1} \stackrel{\delta^{2}}{\leftarrow} \mathrm{C}^{2} \leftarrow 0$ is an exact sequence up to degree 27, where $\mathrm{C}^{1}$ and $\mathrm{C}^{2}$ are free $A$-modules whose bases are $\left\{b_{1}, b_{2}, b_{3}, b_{4}, b_{5}\right\}$ and $\left\{e_{1}, e_{2}, e_{3}, e_{4}\right\}$, with their degrees:

$\operatorname{deg} b_{1}=8, \quad \operatorname{deg} b_{2}=12, \quad \operatorname{deg} b_{3}=14, \quad \operatorname{deg} b_{4}=15, \quad \operatorname{deg} b_{5}=16$, $\operatorname{deg} e_{1}=16, \quad \operatorname{deg} e_{2}=20, \quad \operatorname{deg} e_{3}=22, \quad \operatorname{deg} e_{4}=23$.

$\delta^{1}$ and $\delta^{2}$ are defined as follows:

$$
\begin{aligned}
\delta^{1}\left(b_{1}\right)= & S q(8)+S q(5,1)+S q(2,2)+S q(1,0,1), \\
\delta^{1}\left(b_{2}\right)= & S q(0,4)+S q(6,2)+S q(5,0,1)+S q(2,1,1), \\
\delta^{1}\left(b_{3}\right)= & S q(0,0,2)+S q(5,3)+S q(7,0,1)+S q(4,1,1)+S q(1,2,1), \\
\delta^{1}\left(b_{4}\right)= & S q(0,0,0,1), \\
\delta^{1}\left(b_{5}\right)= & S q(16) \\
\delta^{2}\left(e_{1}\right)= & \{S q(8)+S q(5,1)+S q(2,2)+S q(1,0,1)\} b_{1} \\
& +\{S q(4)+S q(1,1)\} b_{2}+S q(2) b_{3}+S q(1) b_{4}, \\
\delta^{2}\left(e_{2}\right)= & \{S q(0,4)+S q(6,2)+S q(5,0,1)+S q(2,1,1)\} b_{1} \\
& +\{S q(8)+S q(5,1)\} b_{2}+S q(3,1) b_{3}, \\
\delta^{2}\left(e_{3}\right)= & \{S q(0,0,2)+S q(5,3)+S q(7,0,1)+S q(4,1,1)+S q(1,2,1)\} b_{1} \\
& +\{S q(1,3)+S q(3,0,1)+S q(0,1,1)\} b_{2}+\{S q(8)+S q(2,2)\} b_{3}, \\
\delta^{2}\left(e_{4}\right)= & S q(0,0,0,1) b_{1}+\{S q(8)+S q(5,1)+S q(2,2)+S q(1,0,1)\} b_{4} .
\end{aligned}
$$

Proof. We can get them by a routine calculation.

(Q.E. D.)

Theorem 2.4. There is one and only one homotopy type of spectra which realize $\mathcal{A}(2)$ with the $A$-module structure indicated by Mitchell [6].

Proof. We proved $\operatorname{Ext}_{\mathcal{A}}^{s+s+n}\left(\mathcal{A}(2), \boldsymbol{F}_{2}\right) \equiv 0$ for $s \geqq 1,0 \leqq n \leqq 23$. But the fact that $d_{2}\left(h_{2,2}^{\prime}\right)=h_{1,3}^{\prime}$ also implies:

$$
\operatorname{Ext}_{\mathfrak{A}}^{s+1, s+n}\left(\mathcal{A}(2), \boldsymbol{F}_{2}\right) \equiv 0 \quad \text { for } \quad s \geqq 2,0 \leqq n \leqq 23 .
$$

So we must indicate $\operatorname{Ext}_{\mathfrak{l}}^{2,1}(\mathcal{A}(2), \mathcal{A}(2)) \cong 0$, in other words,

$$
\delta^{2 *}: \operatorname{Hom}_{\mathfrak{A}}^{1}\left(\mathrm{C}^{1}, \mathcal{A}(2)\right) \longrightarrow \operatorname{Hom}_{\mathfrak{A}}^{1}\left(\mathrm{C}^{2}, \mathcal{A}^{\prime}(2)\right)
$$

is surjective.

$$
\begin{aligned}
& \operatorname{Hom}_{\mathcal{A}}^{1}\left(\mathrm{C}^{1}, \mathcal{A}(2)\right) \cong \mathcal{A}(2)^{7} \oplus \mathcal{A}(2)^{11} \oplus \mathcal{A}(2)^{13} \oplus \mathcal{A}(2)^{14} \oplus \mathcal{A}(2)^{15} \\
& \operatorname{Hom}_{\mathcal{A}}^{1}\left(\mathrm{C}^{2}, \mathcal{A}(2)\right) \cong \mathcal{A}(2)^{15} \oplus \mathcal{A}(2)^{19} \oplus \mathcal{A}(2)^{21} \oplus \mathcal{A}(2)^{22}
\end{aligned}
$$




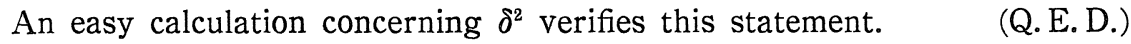

\section{References}

[1] Adams, J.F., On the non-existence of elements of Hopf invariant one, Ann. of Math., 72 (1960), 20-104.

[2] Brown, E.H. and Gitler, S., A spectrum whose cohomology is a certain cyclic module over the Steenrod algebra, Topology, 12 (1973), 283-295.

[3] Davis, D. M. and Mahowald, M., $v_{1-}$ and $v_{2}$ - periodicity in stable homotopy theory, Amer. J. Math., 103 (1981), 615-659.

[4] Lin, W.H., Thesis, Northwestern Univ., 1974.

[5] Milnor, J., The Steenrod algebra and its dual, Ann. of Math., 67 (1958), 150-171.

[6] Mitchell, S. A., Finite complexes with $A(n)$ free cohomology, Topology, 24 (1985), 227-248.

[7] Ravenel, D.C., Complex cobordism and stable homotopy groups of spheres, Academic Press, 121 (1986).

[8] Toda, H., On spectra realizing exterior parts of the Steenrod algebra, Topology, 10 (1971), 53-65. 